\title{
Confocal Laser Endomicroscopy and NSAID Enteropathy: Where Next?
}

\author{
Alastair J. M. Watson
}

Received: 16 March 2014/Accepted: 17 March 2014/Published online: 5 April 2014

(c) Springer Science+Business Media New York 2014

\begin{abstract}
Although Marvin Minsky invented the confocal microscope in 1957, the development of the personal computer in the early 1980s was needed before this invention was widely used in the biological sciences, with commercial confocal endoscopes not available until the first years of the twenty-first century. Confocal microscopy is a method of obtaining sharp images of fluorescence from within solid translucent tissue. Underlying this technique is the fundamental physical observation that when bright light of a particular wavelength (the excitation wavelength) strikes a fluorescent molecule, light of a longer wavelength (the emission wavelength) is emitted. In a confocal microscope, a series of mirrors sweeps a beam of excitation laser light across the tissue in order to restrict excitation to a specific location at any one time. In response to this excitation, fluorescent light emits from the entire depth of the tissue, producing a blurred image. To obtain a sharp image from a single plane within the tissue, a pinhole is positioned in front of the fluorescence detector so that only light from the focal plane within the tissue, termed the confocal point, is transmitted, such that light from above and below the focal plane is excluded. The beauty of this technique is that no physical slicing of the tissue is required as in conventional histology. Instead, the optical sectioning of the confocal microscope creates sharp images from deep within solid tissue. When incorporated into an endoscope, microscopic fluorescent images of the gut mucosa are obtained from
\end{abstract}

A commissioned Editorial for Digestive Diseases and Sciences on Shi et al. [9].

\footnotetext{
A. J. M. Watson $(\bowtie)$

Norwich Medical School, University of East Anglia, Norwich Research Park, Norwich NR4 7TJ, UK

e-mail: alastair.watson@uea.ac.uk
}

beneath the surface. In principle, such images when obtained in loosely structured organs such as the liver can be acquired at depths of $200-300 \mu \mathrm{m}$, although in the intestine the maximum depth is $<50 \mu \mathrm{m}$ due to absorption and diffraction of the emitted light by epithelial nuclei.

To date, confocal endomicroscopy (CLE) has been used in cancer surveillance in inflammatory bowel disease and in Barrett's esophagus due to its ability to target endoscopic pinch biopsies to dysplastic mucosa [1, 2]. Kiesslich et al. reported that the use of CLE targeting increased the proportion of dysplasia-positive biopsies while decreasing the overall number of biopsies collected compared with conventional untargeted biopsy acquisition.

One of the attractions of CLE is that it enables imaging of features not visible with conventional histology. For example, breaches of the epithelial barrier can be imaged as an efflux of intravenous fluorescein into the intestinal lumen at defined cellular sites [3]. Such barrier loss predicts relapse of IBD patients in remission. Intramucosal bacteria can also be imaged by CLE [4]. Both parameters can be obtained in real time during the endoscopic examination without the usual delay inherent in the processing of standard histological specimens.

It has been known for more than 50 years that epithelial cells divide in the crypt and migrate to the tips of the villi in the small intestine or the colonic surface from where they are shed [5]. Until the recent use of confocal microscopy studies of mouse epithelium in vivo, little was known about the shedding process [6], in particular how the intestinal barrier was maintained. During physiological cell shedding, tight junction proteins redistribute around the shedding cell, plugging the gap left after the cell has been extruded [7], maintaining the barrier until cellular migration restores epithelial continuity. Inflammatory stimuli such as tumor necrosis factor (TNF)- $\alpha$ or 
lipopolysaccharide (LPS) increase the number of apoptotic cells within the villous epithelium, with a consequent increase in cell shedding [8]. Redistribution of tight junction proteins still occurs but sometimes fails to seal the resultant gap, particularly when two or more cells are shed simultaneously, producing a gap too large to seal.

It is in this context that Shi et al. [9], in an article published in this issue of Digestive Diseases and Sciences, investigated the effect of indomethacin on the epithelial gaps left by shedding cells in rat small intestine. Using a hand-help rigid confocal endoscope (Optiscan), they were able to visualize the epithelial gaps that occur after cell shedding in exteriorized jejunum. Furthermore, they were able to distinguish epithelial gaps from goblet cells [10]. Administration of indomethacin increased the density of epithelial gaps sevenfold $6 \mathrm{~h}$ later, several hours in advance of ulcer formation, highlighting the exquisite sensitivity of CLE for identifying subtle yet pathophysiologically important mucosal alterations. Two cytoprotective drugs were evaluated for their potential to prevent gap formation; the proton pump inhibitor rabeprazole and geranylgeranylacetone, an ulcer-healing drug whose mode of action remains poorly understood. Both drugs reduced epithelial gap formation, with tissue TNF- $\alpha$ concentrations increasing threefold after administration of indomethacin and reducing to twofold increase after rabeprazole or geranylgeranylacetone. An increase in caspase- 3 could also be demonstrated consistent with increased apoptotic cell shedding after indomethacin. Together, these data suggest that indomethacin increases tissue TNF- $\alpha$, increasing epithelial cell apoptosis, leaving epithelial gaps. Although barrier function integrity of at these sites of cell shedding was not investigated, it might be anticipated that a proportion of these gaps may not be fully sealed, facilitating entry of luminal bacteria, antigens, and toxins, further increasing tissue TNF- $\alpha$ concentrations and the rate cell shedding $[3,11]$. A puzzling feature of the paper was that increased NF- $\mathrm{KB}$ expression was reported following geranylgeranylacetone and rabeprazole treatment, which counters the usual anti-apoptotic and pro-survival effects of $\mathrm{NF}-\kappa \mathrm{B}$. Whether the rise of NF- $\kappa \mathrm{B}$ was protective was not investigated [12]. Overall, this paper adds to our understanding of the toxic effects of indomethacin in the small intestine and demonstrates the striking sensitivity of CLE in demonstrating intestinal pathology not apparent with standard histological techniques.

The future potential of CLE in clinical practice is immense due to the possibility of molecular imaging and is only limited by the availability of targeted fluorescent probes. For example, it is possible to image intestinal epithelial cells expressing membrane-bound TNF (mTNF) with fluorescein isothiocyanate-labeled adalimumab antibodies [13]. Therapeutic antibodies to TNF act by binding to mTNF. Binding to soluble TNF is not therapeutic in Crohn's disease. The authors demonstrated that patients with high numbers of mTNF positive cells have a high response rate to anti-TNF therapy, whereas patients with low numbers of mTNF positive cells do not respond.

Significant obstacles, however, stand in the way of further development of CLE in clinical practice. First, despite the demonstration of its utility in screening for dysplasia in the colon and esophagus, CLE has not been widely adopted in endoscopy units, perhaps due to lack of clarity regarding its advantages over standard practice that would justify its significant expense and substantial training demands. Accordingly, the widespread clinical adoption of CLE awaits demonstration of clinical utility over conventional methods, such as reported by Shi et al. [9], for the detection of early NSAID enteropathy perhaps in patients with occult gastrointestinal blood loss. More importantly, CLE is held back by the lack of fluorescent probes, since fluorescein is the only probe fully licensed for clinical practice, which has substantial drawbacks in that it does not image cellular nuclei well due to its initial clinical development as an extracellular dye [14]. The development of new fluorescent probes for clinical endoscopic use is severely limited by the considerable time and expense required for regulatory approval. Thus, further substantial investment is required for the development of new applications for CLE, which will be accelerated once unique and clinically important indications are identified. In the meantime, the addition of novel applications for fluorescein-based CLE such as the assessment of NSAID enteropathy to the current portfolio of applications including cancer screening and assessment of barrier function will keep CLE alive until fluorescent antibody probes become available and open a new age for realtime molecular endoscopic imaging.

\section{References}

1. Kiesslich R, Burg J, Vieth M, et al. Confocal laser endoscopy for diagnosing intraepithelial neoplasias and colorectal cancer in vivo. Gastroenterology. 2004;127:706-713.

2. Canto MI, Anandasabapathy S, Brugge W, et al. In vivo endomicroscopy improves detection of Barrett's esophagus-related neoplasia: a multicenter international randomized controlled trial (with video). Gastrointest Endosc. 2014;79:211-221.

3. Kiesslich R, Duckworth CA, Moussata D, et al. Local barrier dysfunction identified by confocal laser endomicroscopy predicts relapse in inflammatory bowel disease. Gut. 2012;61:1146-1153.

4. Moussata D, Goetz M, Gloeckner A, et al. Confocal laser endomicroscopy is a new imaging modality for recognition of intramucosal bacteria in inflammatory bowel disease in vivo. Gut. 2011;60:26-33.

5. Eisenhoffer GT, Loftus PD, Yoshigi M, et al. Crowding induces live cell extrusion to maintain homeostatic cell numbers in epithelia. Nature. 2012;484:546-549. 
6. Watson AJ, Hughes KR. TNF-alpha-induced intestinal epithelial cell shedding: implications for intestinal barrier function. Ann NY Acad Sci. 2012;1258:1-8.

7. Marchiando AM, Shen L, Graham WV, et al. The epithelial barrier is maintained by in vivo tight junction expansion during pathologic intestinal epithelial shedding. Gastroenterology. 2011;140:1208.e1-1218.e2.

8. Williams JM, Duckworth CA, Watson AJ, et al. A mouse model of pathological small intestinal epithelial cell apoptosis and shedding induced by systemic administration of lipopolysaccharide. Dis Model Mech. 2013;6:1388-1399.

9. Shi S, Wang $\mathrm{H}, \mathrm{Gao} \mathrm{H}$, et al. Increased gap density predicts weakness of the epithelial barrier in vivo by confocal laser endomicroscopy in indomethacin-induced enteropathy. Dig Dis Sci. (Epub ahead of print). doi:10.1007/s10620-014-3076-8.

10. Kiesslich R, Goetz M, Lammersdorf K, et al. Chromoscopyguided endomicroscopy increases the diagnostic yield of intraepithelial neoplasia in ulcerative colitis. Gastroenterology. 2007; 132:874-882.

11. Watson AJ, Chu S, Sieck L, et al. Epithelial barrier function in vivo is sustained despite gaps in epithelial layers. Gastroenterology. 2005;129:902-912.

12. Nenci A, Becker C, Wullaert A, et al. Epithelial NEMO links innate immunity to chronic intestinal inflammation. Nature. 2007;446:557-561.

13. Atreya R, Neumann $\mathrm{H}$, Neufert $\mathrm{C}$, et al. In vivo imaging using fluorescent antibodies to tumor necrosis factor predicts therapeutic response in Crohn's disease. Nat Med. 2014;20:313-318. doi:10.1038/nm.3462.

14. Wallace MB, Meining A, Canto MI, et al. The safety of intravenous fluorescein for confocal laser endomicroscopy in the gastrointestinal tract. Aliment Pharmacol Ther. 2010;31:548-552. 